\title{
SOLUTIONS OF A GENERALIZED MARKOFF EQUATION IN FIBONACCI NUMBERS
}

\author{
Hayder Raheem Hashim ${ }^{\mathbf{c}}$ - Szabolcs Tengely \\ (Communicated by Milan Paštéka)
}

\begin{abstract}
In this paper, we find all the solutions $(X, Y, Z)=\left(F_{I}, F_{J}, F_{K}\right)$, where $F_{I}, F_{J}$, and $F_{K}$ represent nonzero Fibonacci numbers, satisfying a generalization of Markoff equation called the Jin-Schmidt equation: $A X^{2}+B Y^{2}+C Z^{2}=D X Y Z+1$.

(C) 2020

Mathematical Institute

Mathematical Institute
Slovak Academy of Sciences
\end{abstract}

\section{Introduction}

The Diophantine equation

$$
X^{2}+Y^{2}+Z^{2}=3 X Y Z
$$

in positive integers $X \leq Y \leq Z$ is called the Markoff equation, which was deeply studied by Markoff [11,12] demonstrating a relationship between its integer solutions (so-called Markoff triples) and Diophantine approximation. He obtained many interesting results related to the Markoff triples. He showed that there are infinitely many Markoff triples, which can be generated from the fundamental solution $(1,1,1)$ and the branching operation

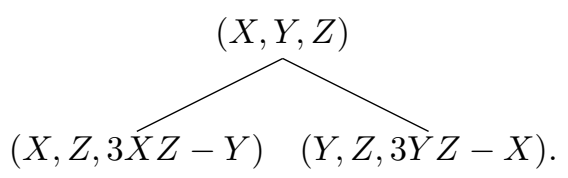

In fact, the set of ordered positive solutions can be organized in a tree called Markoff's tree. A component of some Markoff triple is called a Markoff number. The sequence of Markoff numbers is as follows

$$
1,2,5,13,29,34,89,169,194,233,433,610,985,1325,1597, \ldots
$$

(sequence $A 002559$ [15]) which appear as coordinates of Markoff triples

$$
\begin{aligned}
& (1,1,1),(1,1,2),(1,2,5),(1,5,13),(2,5,29),(1,13,34),(1,34,89),(2,29,169), \\
& (5,13,194),(1,89,233),(5,29,433),(1,233,610),(2,169,985),(13,34,1325), \ldots
\end{aligned}
$$

2010 Mathematics Subject Classification: Primary 11D25; Secondary 11B39.

Keywords: Lucas sequences, Diophantine equations, Markoff equation.

The authors would like to express their sincere gratitude to the referee for the careful reading of the manuscript and many useful comments, which improve the quality of the paper. This work was partially supported by the European Union and the European Social Fund through project EFOP-3.6.1-16-2016-00022 (Sz.T.). The research was supported in part by grantsANN130909, K115479 and K128088 (Sz.T.) of the Hungarian National Foundation for Scientific Research. The work of H. R. Hashim was supported by the Stipendium Hungaricum Scholarship. c Corresponding author. 
This equation has been generalized by several authors. For instance, Hurwitz [8] applied Markoff's descent technique to the equation

$$
X_{1}^{2}+X_{2}^{2}+\cdots+X_{n}^{2}=A X_{1} X_{2} \ldots X_{n}
$$

with $A$ is a nonzero integer and $n \geq 3$. Mordell 13 studied the integer solutions of the equation

$$
X^{2}+Y^{2}+Z^{2}=A X Y Z+B
$$

where $A$ and $B$ are integers with $A>0$. Another generalization was considered by Rosenberger [14, which has the form

$$
A X^{2}+B Y^{2}+C Z^{2}=D X Y Z .
$$

This equation is often called the Markoff-Rosenberger equation. The Markoff-Rosenberger equation was generalized by Jin and Schmidt $\left[9\right.$ in which they determined the solutions $(X, Y, Z) \in \mathbb{N}^{3}$ of the equation

$$
A X^{2}+B Y^{2}+C Z^{2}=D X Y Z+1
$$

Jin and Schmidt showed that equation 1.5 has a fundamental solution if and only if

$$
\begin{aligned}
(A, B, C, D) \in\{ & (2,2,3,6),(2,1,2,2),(7,2,14,14),(3,1,6,6),(6,10,15,30) \\
& (5,1,5,5),(1, b, b, 2 b)\}, \quad \text { with } b \in \mathbb{N} .
\end{aligned}
$$

Other generalizations and studies related to Markoff equation can be found in [3], 5], 2], 7], and the references given there. One of the interesting studies introduced by Luca and Srinivasan [10] in which they found all the triples of Fibonacci numbers $(X, Y, Z)=\left(F_{n_{1}}, F_{n_{2}}, F_{n_{3}}\right)$, with $\bar{F}_{n_{1}} \leq F_{n_{2}} \leq F_{n_{3}}$, satisfying the Markoff equation (1.1). In this paper we investigate the solutions of the Jin-Schmidt equation (1.5) for which $(X, Y, Z)=\left(F_{I}, F_{J}, F_{K}\right)$ in positive integers dropping out the condition that $I \leq J \leq K$. In other words, we study the solutions of the following Diophantine equations in the sequence of Fibonacci numbers

$$
\begin{aligned}
2 X^{2}+2 Y^{2}+3 Z^{2} & =6 X Y Z+1, \\
2 X^{2}+Y^{2}+2 Z^{2} & =2 X Y Z+1, \\
7 X^{2}+2 Y^{2}+14 Z^{2} & =14 X Y Z+1 \\
3 X^{2}+Y^{2}+6 Z^{2} & =6 X Y Z+1 \\
6 X^{2}+10 Y^{2}+15 Z^{2} & =30 X Y Z+1, \\
5 X^{2}+Y^{2}+5 Z^{2} & =5 X Y Z+1 .
\end{aligned}
$$

We remark that the same technique can be applied in case of $(A, B, C, D)=(1, b, b, 2 b)$ for given values of $b$. One of the interesting motivations about the Jin-Schmidt equation is that equation (1.6) appeared in connection with the description of the lower part of the approximation spectrum for quaternions. Moreover, it is connected with the description of approximation constants for complex numbers on the circle $\left\{z \in \mathbb{C}|| z \mid=\frac{1}{\sqrt{2}}\right\}$ with respect to integers in the field $\mathbb{Q}(\sqrt{-3})$. For more details about these connections, one can see e.g. [9] and the references given there.

\section{Auxiliary results}

We introduce some auxiliary results and a procedure that can be used to study the existence and nonexistence of such solutions in equations of the form (1.5) (particularly, equations $1.6-(1.11)$ ). 
Recall that the Binet's Fibonacci numbers formula is known as

$$
F_{n}=\frac{\alpha^{n}-\beta^{n}}{\alpha-\beta} \quad \text { where }(\alpha, \beta)=\left(\frac{1+\sqrt{5}}{2}, \frac{1-\sqrt{5}}{2}\right)
$$

for all $n \geq 0$, where $\alpha$ is called the golden ratio and $\beta=\frac{-1}{\alpha}$. Moreover, it is also known that

$$
\alpha^{n-2} \leq F_{n} \leq \alpha^{n-1} \quad \text { holds for all } n \geq 1 .
$$

\section{The Procedure:}

To start the procedure off, we first have to obtain all the possible distinct equations

$$
a x^{2}+b y^{2}+c z^{2}=d x y z+1
$$

of equation 1.5 by permuting the coefficients $A, B$, and $C$ for

$$
\begin{aligned}
(A, B, C, D) \in S=\{ & (2,2,3,6),(2,1,2,2),(7,2,14,14),(3,1,6,6), \\
& (6,10,15,30),(5,1,5,5)\} .
\end{aligned}
$$

The following steps summarize the technique of investigating all the solutions $(x, y, z)=\left(F_{i}, F_{j}, F_{k}\right)$ with $2 \leq i \leq j \leq k$ for every equation of the form 2.3 for a given tuple $(a, b, c, d)$; that is,

$$
a F_{i}^{2}+b F_{j}^{2}+c F_{k}^{2}=d F_{i} F_{j} F_{k}+1
$$

where $2 \leq i \leq j \leq k$, note that we assumed that $i \geq 2$ since $F_{1}=F_{2}=1$.

- Determining an upper bound for $i$ in equation (2.4). We first rewrite the equation in the form

$$
c F_{k}-d F_{i} F_{j}=-\frac{a F_{i}^{2}+b F_{j}^{2}}{F_{k}}+\frac{1}{F_{k}} .
$$

Inserting the values of $F_{i}, F_{j}$, and $F_{k}$ in the left-hand side of equation 2.5) and using identity (2.1) we obtain that

$$
\frac{c}{\sqrt{5}} \alpha^{k}-\frac{d}{5} \alpha^{i+j}=-\frac{a F_{i}^{2}+b F_{j}^{2}}{F_{k}}+\frac{1}{F_{k}}+\frac{c}{\sqrt{5}} \beta^{k}-\frac{d}{5}\left(\alpha^{i} \beta^{j}+\alpha^{j} \beta^{i}-\beta^{i+j}\right) .
$$

Based on the inequalities 2.2 and $2 \leq i \leq j \leq k$; that is, $1 \leq F_{i} \leq F_{j} \leq F_{k}$, we have that

$$
\begin{aligned}
\frac{a F_{i}^{2}+b F_{j}^{2}}{F_{k}} & \leq(a+b) \frac{F_{j}^{2}}{F_{k}} \leq(a+b) \alpha^{2 j-k} \leq(a+b) \alpha^{j}, \\
\frac{1}{F_{k}} & \leq 1<\alpha^{j}, \\
\left|\frac{c}{\sqrt{5}} \beta^{k}\right| & =\left|-\frac{c}{\sqrt{5}} \alpha^{-k}\right| \leq \frac{c}{\sqrt{5}} \alpha^{-j} \leq \frac{c}{5} \alpha^{j}, \\
\left|\frac{d}{5}\left(\alpha^{i} \beta^{j}+\alpha^{j} \beta^{i}-\beta^{i+j}\right)\right| & \leq \frac{d}{5}\left(2 \alpha^{j}+1\right) \leq \frac{3 d}{5} \alpha^{j} .
\end{aligned}
$$

Taking the absolute values to equation $(2.6)$ and using the inequalities 2.7$)-(2.10)$ we obtain that

$$
\left|\frac{c}{\sqrt{5}} \alpha^{k}-\frac{d}{5} \alpha^{i+j}\right|<\left(1+a+b+\frac{c+3 d}{5}\right) \alpha^{j} .
$$

Multiplying across by $\frac{\sqrt{5}}{c \alpha^{i+j}}$, we get that

$$
\left|\alpha^{k-i-j}-\frac{d}{c \sqrt{5}}\right|<\frac{h}{\alpha^{i}},
$$


where $h=\frac{\sqrt{5}}{c}\left(1+a+b+\frac{c+3 d}{5}\right)$. Suppose that

$$
\min _{n \in \mathbb{Z}}\left|\alpha^{n}-\frac{d}{c \sqrt{5}}\right|>g>0,
$$

so inequality 2.11 implies that

which clearly gives

$$
g<\frac{h}{\alpha^{i}}
$$

$$
i \leq\left\lfloor\frac{\ln \left(\frac{h}{g}\right)}{\ln (\alpha)}\right\rfloor=l .
$$

- Determining an upper bound for $k-j$ in equation (2.4). We provide such a bound using the upper bound for $i$ ( that is, $i \leq l$ ) and inequality (2.11). We have that $1 \leq a, b, c \leq 15$ and $2 \leq d=D \leq 30$, which imply that $h \leq 52 \sqrt{5}<116.3$. Therefore, inequality (2.11) becomes

$$
|| \alpha^{k-i-j}|-| \frac{d}{c \sqrt{5}}|| \leq\left|\alpha^{k-i-j}-\frac{d}{c \sqrt{5}}\right|<\frac{116.3}{\alpha^{2}}<44.5 \quad \text { as } i \geq 2,
$$

which leads to

$$
\left|\alpha^{k-i-j}\right|<44.5+\left|\frac{d}{c \sqrt{5}}\right|<44.5+\frac{30}{\sqrt{5}}<58 \quad \text { as } d \leq 30 \text { and } c \geq 1 .
$$

Hence,

$$
k-j<i+\frac{\ln (58)}{\ln (\alpha)}<l+9 \quad \text { or } \quad k \leq j+l+8 \quad \text { as } i \leq l .
$$

- Eliminating the values of $i$ for $i \in[2, l]$ in which equation (2.4) does not hold (and then equation 2.3 for which $(x, y, z)=\left(F_{i}, F_{j}, F_{k}\right)$ with $\left.2 \leq i \leq j \leq k\right)$. For that, we solve the Diophantine equation

$$
a F_{i}^{2}+b y^{2}+c z^{2}-d F_{i} y z-1=0
$$

for $y$ and $z$. This can be done by SageMath [16] using the function solve_diophantine(). If there exists no $i$ for which equation 2.14 is satisfied, then equation (2.3) does not have any solution $(x, y, z)=\left(F_{i}, F_{j}, F_{k}\right)$ with $2 \leq i \leq j \leq k$ at the tuple $(a, b, c, d)$.

- Fixing $i$ and $k$ for an arbitrary $k \in\{j, j+1, \ldots, j+l+8\}$ in equation 2.4 we get that

$$
b F_{j}^{2}-s F_{j}+w=0,
$$

where $s=d F_{i} F_{k}$ and $w=a F_{i}^{2}+c F_{k}^{2}-1$. We note that the equation above only depends on $j$ for all $j \geq i \geq 2$.

- Determining whether there exists $j$ for which equation 2.15) holds using any of the following arguments.

(i) The technique of using the quadratic formula and the identity relationship between the Fibonacci numbers and Lucas numbers. The sequence of Lucas numbers $\left\{L_{n}\right\}$ is defined by $L_{0}=2, L_{1}=1$ and $L_{n}=L_{n-1}+L_{n-2}$, for $n \geq 2$. Terms of the Fibonacci sequences and Lucas sequences satisfy the identity

$$
L_{k}^{2}=5 F_{k}^{2} \pm 4 .
$$

Multiplying 2.15 by $4 b$ and adding $s^{2}$ to both sides lead to

$$
\left(2 b F_{j}-s\right)^{2}=s^{2}-4 b w .
$$

Multiplying equations (2.16) and 2.17) together yields

$$
Y_{1}^{2}=\left(5 X_{1}^{2} \pm 4\right)\left(d^{2} F_{i}^{2} X_{1}^{2}-4 b\left(a F_{i}^{2}+c X_{1}^{2}-1\right)\right),
$$




\section{SOLUTIONS OF A GENERALIZED MARKOFF EQUATION IN FIBONACCI NUMBERS}

where $X_{1}=F_{k}$ and $Y_{1}=L_{k}\left(2 b F_{j}-d F_{i} F_{k}\right)$. Therefore, our problem is reduced to obtain integral points on these biquadratic genus 1 curves. This will be realized using an algorithm implemented in Magma 4 as SIntegralLjunggrenPoints() or an algorithm described by Alekseyev and Tengely [1] in which they gave an algorithmic reduction of the search for integral points on such a curve to solving a finite number of Thue equations.

(ii) The Fibonacci identities substitution technique in which we use the Fibonacci sequence formula or some related identities to eliminate equation (2.15).

(iii) The congruence argument technique in which we eliminate equation 2.15) modulo a prime number $p$.

Applications of these arguments will be shown in details in the proof of Theorem 3.1 .

- From every obtained solution $(x, y, z)=\left(F_{i}, F_{j}, F_{k}\right)$ of equation $(2.3)$ at the tuple $(a, b, c, d)$ we derive the corresponding solution $(X, Y, Z)=\left(F_{I}, F_{J}, F_{K}\right)$ of equation 1.5$)$ at the tuple $(A, B, C, D)$ by comparing the positions of the components of their tuples.

\section{Main results}

THEOREM 3.1. Let $m$ be a positive integer greater than 1 . If $(X, Y, Z)=\left(F_{I}, F_{J}, F_{K}\right)$ is a solution of equation (1.5) with $(A, B, C, D) \in S$, then the complete list of solutions is given by

\begin{tabular}{|c|c|c|}
\hline$E q$. & $(A, B, C, D)$ & $\{(X, Y, Z)\}$ \\
\hline \hline 1.6 & $(2,2,3,6)$ & $\left\{(1,1,1),(1,2,1),(1,2,3),(2,1,1),(2,1,3),\left(F_{2 m-1}, F_{2 m+1}, 1\right),\left(F_{2 m+1}, F_{2 m-1}, 1\right)\right\}$ \\
\hline 1.7 & $(2,1,2,2)$ & $\{(2,3,2),(2,5,2),(2,5,8),(8,5,2)\}$ \\
\hline 1.8 & $(7,2,14,14)$ & $\{(1,2,1),(1,5,1),(3,2,1),(3,2,5)\}$ \\
\hline 1.9 & $(3,1,6,6)$ & $\{(1,2,1),(3,2,1),(3,2,5)\}$ \\
\hline 1.10 & $(6,10,15,30)$ & $\{(1,1,1),(1,2,1),(1,2,3)\}$ \\
\hline 1.11 & $(5,1,5,5)$ & \{\} \\
\hline
\end{tabular}

Pro of of Theorem 3.1. We follow the procedure to obtain and prove the desired solutions in the theorem.

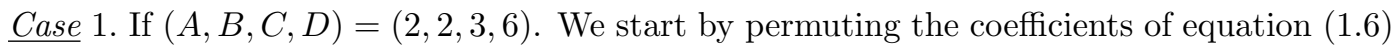
to obtain the equations

$$
\begin{aligned}
& 2 x^{2}+2 y^{2}+3 z^{2}=6 x y z+1, \\
& 2 x^{2}+3 y^{2}+2 z^{2}=6 x y z+1, \\
& 3 x^{2}+2 y^{2}+2 z^{2}=6 x y z+1 .
\end{aligned}
$$

We investigate the solutions of these equations with the assumptions of $x=F_{i}, y=F_{j}$, and $z=F_{k}$ for $2 \leq i \leq j \leq k$. That will be pursued by determining upper bounds for $i$ and $k-j$ as described in the procedure. Thus, we have that the upper bounds for $i$ in equations $\sqrt{3.1},(3.2)$, and $\sqrt{3.3})$ are given by $i \leq 8, i \leq 7$, and $i \leq 7$, respectively. Therefore, we get that $k-j \leq 16, k-j \leq 15$, and $k-j \leq 15$ in case of equations (3.1), (3.2), and (3.3), respectively. Let us consider equation (3.1) with $(x, y, z)=\left(F_{i}, F_{j}, F_{k}\right)$; that is, $2 F_{i}^{2}+2 F_{j}^{2}+3 F_{k}^{2}-6 F_{i} F_{j} F_{k}-1=0$ with $2 \leq i \leq j \leq k$ such that $2 \leq i \leq 8$ and $k \leq j+16$. Here, we obtain that $i \in\{2,3,5,7\}$ in which the equation $2 F_{i}^{2}+2 y^{2}+3 z^{2}-6 F_{i} y z-1=0$ is solvable. For $i=2$ and $k \in\{j, j+1, \ldots, j+16\}$, we have that

$$
2 F_{j}^{2}-6 F_{k} F_{j}+3 F_{k}^{2}+1=0 .
$$

For each $k$ we determine the values of $j$ in which the latter equation is satisfied, we consider the equation as a quadratic in $F_{j}$ and follow the argument described in (i) with $(a, b, c, d)=(2,2,3,6)$. 
It remains to solve the quartic Diophantine equations

$$
\begin{aligned}
& Y_{1}^{2}=60 X_{1}^{4}+8 X_{1}^{2}-32, \\
& Y_{1}^{2}=60 X_{1}^{4}-88 X_{1}^{2}+32,
\end{aligned}
$$

where $X_{1}=F_{k}$, and $Y_{1}=\left(4 F_{j}-6 F_{k}\right) L_{k}$. The integral points on these equations can be obtained using the Magma function SIntegralLjunggrenPoints(). For equation (3.5) we get that $X_{1} \in$ $\{ \pm 1, \pm 3\}$. In case of equation (3.6) we have that $X_{1} \in\{ \pm 1\}$. It follows that $F_{k}=1$ or 3 . Hence,

- If $k=j$ and $F_{k}=1$, then we have that $k=j=2$. Therefore, we get the solution $(x, y, z)=$ $\left(F_{2}, F_{2}, F_{2}\right)=(1,1,1)$.

- If $k \in[j+1, j+16]$, then $F_{k}=1$ is impossible for all $j \geq 2$.

- If $k=j$ and $F_{k}=3$, then we obtain that $k=j=4$. However, the triple $(1,3,3)$ is clearly not a solution to equation 3.1.

- If $k=j+1$ and $F_{k}=3$, then we get that $k=4$ and $j=3$. Thus, we have the solution $(x, y, z)=(1,2,3)$.

- If $k=j+2$ and $F_{k}=3$, then we obtain that $k=4$ and $j=2$. Again, we have that $(x, y, z)=$ $(1,1,3)$ is not a solution to equation (3.1).

- Finally, we have that $F_{k}=3$ is impossible for all $k \in\{j+3, \ldots, j+16\}$ with $j \geq 2$.

Now, we deal with the case of $i=3$ and $k \in[j, j+16]$ with $j \geq 3$. In a similar way, we follow the same argument on the following quadratic equation in $F_{j}$ :

$$
2 F_{j}^{2}-12 F_{k} F_{j}+3 F_{k}^{2}+7=0 .
$$

We obtain the equations

$$
Y_{1}^{2}=600 X_{1}^{4}+200 X_{1}^{2}-224 \quad \text { and } \quad Y_{1}^{2}=600 X_{1}^{4}-760 X_{1}^{2}+224,
$$

with $X_{1}=F_{k}$ and $Y_{1}=\left(4 F_{j}-12 F_{k}\right) L_{k}$. In the former equation we get that $X_{1} \in\{ \pm 1, \pm 3\}$. In the latter equation we obtain that $X_{1} \in\{ \pm 1\}$. It follows that $F_{k}=1$ or 3 , which leads to no solution of equation (3.1). Furthermore, using the same argument described in (i) one can show that equation (3.1) does not have any solution of the form $(x, y, z)=\left(F_{i}, F_{j}, F_{k}\right)$ with $i \leq j \leq k$ in case the of $i=5$ or 7 and $k \in[j, j+16]$.

Next, for equation $(3.2)$ we study the equation $2 F_{i}^{2}+3 F_{j}^{2}+2 F_{k}^{2}-6 F_{i} F_{j} F_{k}-1=0$ with 2 $\leq i \leq 7$ and $j \leq k \leq j+15$. Again, we get that $i \in\{2,3,5,7\}$ in which the equation $2 F_{i}^{2}+3 y^{2}+$ $2 z^{2}-6 F_{i} y z-1=0$ is solvable in $y$ and $z$. Starting with $i=2$ and $k \in\{j, j+1, \ldots, j+15\}$ and following the technique (i), we obtain that $F_{k} \in\{1,2\}$. Therefore:

- If $k=j$ and $F_{k}=1$, we get the solution $(x, y, z)=(1,1,1)$.

- If $j+1 \leq k \leq j+15$, then $F_{k}=1$ is impossible for all $j \geq 2$.

- If $k=j$ and $F_{k}=2$, we obtain that $(x, y, z)=(1,2,2)$, which is not a solution to equation (3.2.

- If $k=j+1$ and $F_{k}=2$, we have the solution $(x, y, z)=(1,1,2)$.

- If $F_{k}=2$ and $k \in\{j+2, \ldots, j+15\}$ for $j \geq 2$, then we get no solution to equation (3.2).

Using the same argument one can prove that the remaining values of $i$ do not lead to any solution of equation 3.2 .

Finally, we deal with equation (3.3) in which we have that $2 \leq i \leq 7$ and $j \leq k \leq j+15$. Here, we get that the equation $3 F_{i}^{2}+2 y^{2}+2 z^{2}-6 F_{i} y z-1=0$ is solvable only at $i \in\{2,4\}$. If $i=2$, 
then the equation $3 F_{i}^{2}+2 F_{j}^{2}+2 F_{k}^{2}-6 F_{i} F_{j} F_{k}-1=0$ becomes

$$
F_{j}^{2}-3 F_{k} F_{j}+F_{k}^{2}+1=0
$$

where $k \in[j, j+15]$ and $j \geq 2$. We obtain the solutions of equation (3.8) (and then of equation (3.3) in case of $i=2$ ) by direct substitutions of the values of $k$ in the equation or using the arguments (ii) and (iii). Thus:

- If $k=j$, then equation (3.8) implies that $F_{j}=1$ and we get the solution $(x, y, z)=(1,1,1)$.

- If $k=j+1$, we claim that $(x, y, z)=\left(F_{2}, F_{2}, F_{3}\right)=(1,1,2)$ is the only solution to equation (3.3). To prove our claim, we must show that equation 3.8 does not hold at $k=j+1$ for all $j \geq 3$. In other words, we follow the idea described in the argument (ii) by using the sequence of Fibonacci numbers ( that is, $F_{j}=F_{j-1}+F_{j-2}$ for $j>2$ ) to show that $F_{j}^{2}-3 F_{j} F_{j+1}+F_{j+1}^{2}+1<0$ for all $j \geq 3$. Hence, let us start with the left-hand side,

$$
\begin{aligned}
F_{j}^{2}-3 F_{j} F_{j+1}+F_{j+1}^{2}+1 & =F_{j}^{2}-3 F_{j}\left(F_{j-1}+F_{j}\right)+\left(F_{j-1}+F_{j}\right)^{2}+1 \\
& =-F_{j}^{2}-F_{j-1} F_{j}+F_{j-1}^{2}+1 \\
& =-F_{j}^{2}-F_{j-2} F_{j-1}+1<0 \quad \text { for all } j \geq 3,
\end{aligned}
$$

and this proves the claim.

- If $k=j+2$, then equation (3.8) gives us the identity

$$
F_{j}^{2}-3 F_{j} F_{j+2}+F_{j+2}^{2}+1=0,
$$

which is valid for all $j=2 m-1$ with $m \geq 2$. This identity was proven in 2018 by Hoare G. 6]. Therefore, we get the solution $(x, y, z)=\left(F_{2}, F_{j}, F_{k}\right)=\left(1, F_{2 m-1}, F_{2 m+1}\right)$.

- If $k=j+3$, then in a similar way of the technique described in 3.9 we can show that the equation $F_{j}^{2}-3 F_{j} F_{j+3}+F_{j+3}^{2}+1>0$ for all $j \geq 2$. That is

$$
F_{j}^{2}-3 F_{j} F_{j+3}+F_{j+3}^{2}+1=F_{j} F_{j+1}+2 F_{j-1} F_{j}+3 F_{j-1} F_{j+1}+F_{j-1}^{2}+1>0
$$

for all $j \geq 2$.

- Similarly, if $k=j+4$ or $j+5$, then we conclude the unsolvability of equation (3.8) by having that

$$
F_{j}^{2}-3 F_{j} F_{j+4}+F_{j+4}^{2}+1=2 F_{j-1} F_{j+2}+F_{j-1} F_{j+3}+F_{j}^{2}+F_{j+1}^{2}+F_{j+3}^{2}+1>0
$$

for all $j \geq 2$, or

$$
F_{j}^{2}-3 F_{j} F_{j+5}+F_{j+5}^{2}+1=F_{j}^{2}+F_{j+3}^{2}+F_{j+4}^{2}+3 F_{j} F_{j+2}+4 F_{j-1} F_{j+4}+1>0
$$

for all $j \geq 2$, respectively.

- If $k=j+6$, we have the equation, call it, $G_{j}=F_{j}^{2}-3 F_{j} F_{j+6}+F_{j+6}^{2}+1=0$. Here, we may use a congruence argument to show the given equation does not hold for all $j \geq 2$ by finding a prime number $p$ in which $G_{j} \not \equiv 0(\bmod p)$. Let $p=17$, then for all $j \geq 2$ we have that

$$
G_{j}(\bmod 17): \equiv 6,5,7,16,5,16,7,5,6,13,14,12,3,14,3,12,14,13,6,5,7, \ldots,
$$

which clearly has period $=18$. This contradicts that $G_{j \geq 2}=0$. Hence, equation (3.3) has no solutions of the form $(x, y, z)=\left(F_{i}, F_{j}, F_{k}\right)$ where $i=2, j \geq 2$, and $k=j+6$.

- Similarly, if $k=j+7$ and $p=13$, then we get that $F_{j}^{2}-3 F_{j} F_{j+7}+F_{j+7}^{2}+1(\bmod 13): \equiv$

$$
3,9,6,12,12,1,12,12,6,9,3,3,1,3,3,9,6,12,12, \ldots \quad \text { for all } j \geq 2 \text {. }
$$

Again, we get a contradiction. For the remaining values of $k$ one can show that equation (3.3) has no more solutions using either of the arguments described in (ii) or (iii). Finally, we deal with $i=4$ that leads to $F_{j}^{2}-9 F_{j} F_{k}+F_{k}^{2}+13=0$. Using the argument described in 
(i), we get that $F_{k}=1$ or 2 , which is impossible for all $k \in\{j, j+1, j+2, \ldots, j+15\}$ with $j \geq 4$.

Finally, we combine the solutions of equations $3.1,(3.2)$, and 3.3 and then permute their components in which they satisfy equation (1.6). Therefore, equation (1.6) has the following set of solutions

$$
\begin{aligned}
(X, Y, Z)= & \left(F_{I}, F_{J}, F_{K}\right) \in\{(1,1,1),(1,2,1),(1,2,3),(2,1,1),(2,1,3), \\
& \left.\left(F_{2 m-1}, F_{2 m+1}, 1\right),\left(F_{2 m+1}, F_{2 m-1}, 1\right)\right\} \quad \forall \quad m \geq 2 .
\end{aligned}
$$

Case 2. If $(A, B, C, D)=(2,1,2,2)$. Again, by permuting the coefficients of equation (1.7) we obtain the distinct equations

$$
\begin{aligned}
& 2 x^{2}+y^{2}+2 z^{2}=2 x y z+1, \\
& x^{2}+2 y^{2}+2 z^{2}=2 x y z+1, \\
& 2 x^{2}+2 y^{2}+z^{2}=2 x y z+1 .
\end{aligned}
$$

To study the solutions of these equations for which $x=F_{i}, y=F_{j}$, and $z=F_{k}$ with $2 \leq i \leq$ $j \leq k$, we compute the upper bounds for $i$ and $k-j$ using the inequalities (2.12) and (2.13), respectively. It follows that the upper bounds for $i$ in equations $(3.10),(3.11)$, and $(3.12)$ are presented by $i \leq 9, i \leq 9$, and $i \leq 10$, respectively. Therefore, we get that $k-j \leq 17$ in equations (3.10) and (3.11) and $k-j \leq 18$ in equation (3.12). Moreover, we have that the equations $2 F_{i}^{2}+y^{2}+2 z^{2}-2 F_{i} y z-1=0$ with $2 \leq i \leq 9$ and $2 F_{i}^{2}+2 y^{2}+z^{2}-2 F_{i} y z-1=0$ with $2 \leq i \leq 10$ are solvable only for $i=3$ or 6 . On the other hand, the equation $F_{i}^{2}+2 y^{2}+2 z^{2}-2 F_{i} y z-1=0$ with $2 \leq i \leq 9$ is solvable for $i \in\{2,4,5,7\}$. As a result of using the argument described in (i), we obtain that the solutions of equation 3.10 and equation 3.12 are given by $(x, y, z)=$ $(2,5,8)$ and $(x, y, z) \in\{(2,2,3),(2,2,5)\}$, respectively. On the other hand, we have no solution to equation (3.11). From these solution, we conclude that the solutions of equation (1.7) are given by $(X, Y, Z)=(2,3,2),(2,5,2),(2,5,8)$, and $(8,5,2)$.

$\underline{\text { Case }}$ 3. If $(A, B, C, D)=(7,2,14,14)$. In a similar way, from equation 1.8 we get the equations

$$
\begin{aligned}
& 7 x^{2}+2 y^{2}+14 z^{2}=14 x y z+1, \\
& 2 x^{2}+7 y^{2}+14 z^{2}=14 x y z+1, \\
& 7 x^{2}+14 y^{2}+2 z^{2}=14 x y z+1, \\
& 14 x^{2}+7 y^{2}+2 z^{2}=14 x y z+1, \\
& 14 x^{2}+2 y^{2}+7 z^{2}=14 x y z+1, \\
& 2 x^{2}+14 y^{2}+7 z^{2}=14 x y z+1 .
\end{aligned}
$$

Assuming that $x=F_{i}, y=F_{j}$, and $z=F_{k}$ with $2 \leq i \leq j \leq k$, we get that $i \leq 8$ and $i \leq 9$ in which the equations (3.13)- -3.16$)$ and equations 3.17$)-(3.18)$ can be held, respectively. Hence, the upper bounds for $k-j$ in equations (3.13)-(3.16) and (3.17)-(3.18) are given by $k-j \leq 16$ and $k-j \leq 17$, respectively. Eliminating some of the values of $i$ in the given ranges, we get that $i \in\{2,4\}$, $i \in\{3,5\}$, and $i \in\{2,5\}$ in which equations; (3.13) and (3.15), (3.14) and (3.18), and (3.16) and (3.17) for which $x=F_{i}$ are solvable, respectively. As before we use the argument described in (i) to get the solution $(x, y, z)=(2,3,5)$ to equation (3.14) and the solutions $(x, y, z)=(1,1,2)$ and $(1,1,5)$ to equations (3.15) and (3.16). Moreover, the equation (3.17) has no more solution other than $(x, y, z)=(1,2,3)$. The remaining equations have no such solutions. These solutions yield the solutions of $(1.8)$, which are given by $(X, Y, Z) \in\{(1,2,1),(1,5,1),(3,2,1),(3,2,5)\}$. 
Case 4 . If $(A, B, C, D)=(3,1,6,6)$. By permuting the coefficients of equation 1.9$)$ we have the following distinct equations

$$
\begin{aligned}
& 3 x^{2}+y^{2}+6 z^{2}=6 x y z+1, \\
& x^{2}+3 y^{2}+6 z^{2}=6 x y z+1, \\
& x^{2}+6 y^{2}+3 z^{2}=6 x y z+1, \\
& 6 x^{2}+y^{2}+3 z^{2}=6 x y z+1, \\
& 6 x^{2}+3 y^{2}+z^{2}=6 x y z+1, \\
& 3 x^{2}+6 y^{2}+z^{2}=6 x y z+1 .
\end{aligned}
$$

Suppose that $x=F_{i}, y=F_{j}$, and $z=F_{k}$ with $2 \leq i \leq j \leq k$. As before we obtain that $i \leq 8$, $i \leq 9$, and $i \leq 12$ for which equations $(3.19)-(3.20),(3.21)-(3.22)$, and $(3.23)-(3.24)$ can be valid, respectively. The upper bounds of $k-j$ can be easily followed using inequality (2.13). Furthermore, we get that $i \in\{2,4\}, i \in\{2,3\}, i \in\{2,4,5\}$, and $i \in\{2,4,5,11\}$ in which the equations; (3.19) and (3.24), 3.20) and (3.21), (3.22), and (3.23) are solvable in $y$ and $z$ for all $x=F_{i}$, respectively. It remains to study the solutions of these equation using any of the arguments described in (i), (ii), or (iii), we obtain that the only solution for equation (3.20) is $(x, y, z)=(2,3,5)$ and for equation $(3.22)$ is $(x, y, z)=(1,2,3)$. Moreover, equations (3.23) and (3.24) have no more solutions other than $(x, y, z)=(1,1,2)$. Finally, the remaining equations are unsolvable. Therefore, the solutions of the main equation (1.9) are given by $(X, Y, Z)=(1,2,1),(3,2,1)$, and $(3,2,5)$.

$\underline{\text { Case }} 5$. If $(A, B, C, D)=(6,10,15,30)$. Permuting the coefficients of equation 1.10$)$ gives the equations

$$
\begin{aligned}
& 6 x^{2}+10 y^{2}+15 z^{2}=30 x y z+1, \\
& 10 x^{2}+6 y^{2}+15 z^{2}=30 x y z+1, \\
& 6 x^{2}+15 y^{2}+10 z^{2}=30 x y z+1, \\
& 15 x^{2}+6 y^{2}+10 z^{2}=30 x y z+1, \\
& 10 x^{2}+15 y^{2}+6 z^{2}=30 x y z+1, \\
& 15 x^{2}+10 y^{2}+6 z^{2}=30 x y z+1 .
\end{aligned}
$$

Let $x=F_{i}, y=F_{j}$, and $z=F_{k}$ with $2 \leq i \leq j \leq k$. Here we get that $i \leq 8$ and $i \leq 7$ for which equations $(3.25)-(3.26)$ and $(3.27)-(3.30)$ can be solvable, respectively. Therefore, $k \leq$ $j+16$ in equations (3.25)-(3.26) and $k \leq j+15$ in the equations (3.27)-(3.30). Eliminating some of these values of $i$ we obtain that $i \in\{2\}, i \in\{2,3\}$, and $i \in\{2,4\}$ for which equations; (3.25) and (3.27), (3.26) and (3.29), and (3.28) and (3.30) are satisfied for all $x=F_{i}$. Using any of the mentioned arguments mainly the one described in (i) we get that $(x, y, z)=(1,1,1)$ and $(1,2,3)$ are the solutions for equation 3.25 and $(x, y, z)=(1,1,1)$ is the only solution for equations (3.26), (3.29), and (3.30). The solutions of equations (3.27) and 3.28$)$ are given by $(x, y, z)=(1,1,1)$ and $(1,1,2)$. Combining these solutions back to equation (1.10) we get that $(X, Y, Z) \in\{(1,1,1),(1,2,1),(1,2,3)\}$.

Case 6 . If $(A, B, C, D)=(5,1,5,5)$. In a similar way, we investigate the solutions of the equations

$$
\begin{aligned}
& 5 x^{2}+y^{2}+5 z^{2}=5 x y z+1, \\
& x^{2}+5 y^{2}+5 z^{2}=5 x y z+1, \\
& 5 x^{2}+5 y^{2}+z^{2}=5 x y z+1,
\end{aligned}
$$


where $x=F_{i}, y=F_{j}$, and $z=F_{k}$ with $2 \leq i \leq j \leq k$. The upper bounds for $i$ in which the given equations can be held are given by $i \leq 8$ and $i \leq 9$ in equations (3.31)-(3.32 and (3.33), respectively. These imply that $k-j \leq 16$ and $k-j \leq 17$ in equations (3.31)-(3.32) and (3.33), respectively. Furthermore, we get that $i \in\{2,3\}$ and $i \in\{2\}$ for which equations; (3.31) and (3.33), and (3.32) with $x=F_{i}$ are solvable in $y$ and $z$, respectively. Using any of the arguments described in (i), (ii), or (iii) leads to the unsolvability of equations (3.31)- 3.33) for which $(x, y, z)=\left(F_{i}, F_{j}, F_{k}\right)$. Therefore, equation (1.11) has no solution of the form $(X, Y, Z)=\left(F_{I}, F_{J}, F_{K}\right)$ in positive integers. Hence, Theorem 3.1 is completely proved.

\section{REFERENCES}

[1] ALEKSEYEV, M. A.-TENGELY, SZ.: On integral points on biquadratic curves and near-multiples of squares in Lucas sequences, J. Integer Seq. 17(6) (2014), Art. ID 14.6.6.

[2] BAER, C.-ROSENBERGER, G.: The equation $a x^{2}+b y^{2}+c z^{2}=d x y z$ over quadratic imaginary fields, Results Math. 33(1-2) (1998), 30-39.

[3] BARAGAR, A.-UMEDA, K.: The asymptotic growth of integer solutions to the Rosenberger equations, Bull. Aust. Math. Soc. 69(3) (2004), 481-497.

[4] BOSMA, W.-CANNON, J.-PLAYOUST, C.: The Magma algebra system. I. The user language, J. Symbolic Comput. 24(3-4) (1997), 235-265.

[5] GONZÁLEZ-JIMÉNEZ, E.: Markoff-Rosenberger triples in geometric progression, Acta Math. Hung. 142(1) (2014), 231-243.

[6] HOARE, G. T. Q.: 102.37 Markov numbers, The Mathematical Gazette 102(555) (2018), 494-496.

[7] HU, S.-LI, Y.: The number of solutions of generalized Markoff-Hurwitz-type equations over finite fields, J. Zhejiang Univ., Sci. Ed. 44(5) (2017), 516-519, 537.

[8] HURWITZ, A.: Über eine Aufgabe der unbestimmten Analyse, Arch. der Math. u. Phys. 11(3) (1907), $185-196$.

[9] JIN, Y.-SCHMIDT, A. L.: A Diophantine equation appearing in Diophantine approximation, Indag. Math., New Ser. 12(4) (2001), 477-482.

[10] LUCA, F.-SRINIVASAN, A.: Markov equation with Fibonacci components, Fibonacci Quart. 56(2) (2018), $126-129$.

[11] MARKOFF, A. A.: Sur les formes quadratiques binaires indéfinies, Math. Ann. 15 (1879), 381-407.

[12] MARKOFF, A. A.: Sur les formes quadratiques binaires indéfinies, Math. Ann. 17 (1880), 379-400.

[13] MORDELL, L. J.: On the integer solutions of the equation $x^{2}+y^{2}+z^{2}+2 x y z=n$, J. Lond. Math. Soc. 28 (1953), 500-510.

[14] ROSENBERGER, G.: Über die Diophantische Gleichung $a x^{2}+b y^{2}+c z^{2}=d x y z$, J. Reine Angew. Math. 305 (1979), 122-125.

[15] SLOANE, N. J. A.-CONWAY, J. H.: The On-Line Encyclopedia of Integer Sequences, https://oeis.org/ A002559

[16] STEIN, W. A. et al.: Sage Mathematics Software (Version 9.0), The Sage Development Team (2020), http: //www.sagemath.org

Received 19. 10. 2019

Accepted 5. 2. 2020
Institute of Mathematics

University of Debrecen

P. O. Box 400

4002 Debrecen

HUNGARY

E-mail: hashim.hayder.raheem@science.unideb.hu tengely@science.unideb.hu 